and complex-characteristics which provide considerable opportunity for disagreement. On the other hand, there are some things that can be said with little fear of contradiction. Presumably few people would now object to, for example, the general definition of the asthenosphere as a layer with no enduring resistance to a shearing stress and thus with a finite strength small enough to be neglected (Le Pichon et al., Plate Tectonics, Elsevier, 1973). It is interesting to note, however, that modern as this difinition may sound, the possibility of flow inherent in it had actually been implied by the acceptance of the principle of isostasy more than a century ago; and it was the problem of isostasy which later led Barrel (J. Geol., 22, 441 and 655; 1914) to incorporate the flow property into a layer model involving a 'lithosphere' and an 'asthenosphere'. In other words, by a curious coincidence the concept of an asthenospheric layer came about independently at almost exactly the time that Wegener was proposing continental drift, the phenomenon that would later require the characteristics of the asthenosphere to provide a convincing mechanism.

But it was to be a long time before the asthenosphere would be properly defined in non-isostatic terms, even though the process began with Gutenberg's early suggestion ( $Z$. Geophys., 2, 24; 1926) that the general monotonic increase in seismic velocity with depth in the Earth may not be applicable to the uppermost mantle. Much later, in the light of many more and more modern seismic investigations, Gutenberg (Physics of the Earth's Interior, Academic Press, 1959) was able to propose the existence of an upper mantle low velocity zone having a minimum $P$ wave velocity at a depth of about $100 \mathrm{~km}$ and a minimum $S$ wave velocity within the depth range $100-200 \mathrm{~km}$, the maximum decrease in velocity being a few percent for each type of wave but slightly greater for $S$ waves than $P$ waves. It now seems doubtful whether the $\mathrm{P}$ wave channel is universal, but the more pronounced $S$ wave low velocity zone has been confirmed. It probably begins at a depth of $70-80 \mathrm{~km}$ beneath typical ocean basins and in the range 110-130 km beneath continental shields, in each case ending at a depth of about $250 \mathrm{~km}$ (Press, J. geophys. Res. $\mathbf{7 5}$, $6575 ; 1970)$. The viscosity in the vicinity of this zone is also apparently low, both Gordon (Geophys. J., 14, 33; 1967) and McKenzie (Geophys. J., 14, 297: 1967) having estimated a value of about $10^{21}$ poise which thereafter rises very rapidly with depth. In summary, then, the asthenosphere seems to be a relatively thin upper mantle layer which varies somewhat in depth and thickness according to the tectonic environment above, in which seismic velocity is anomalously low (and seismic attenuation high), and in which viscosity is also particularly low.

But how does this peculiar combination of properties arise? According to Ringwood (in The Earth's Crust and Upper Mantle, American Geophysical Union, 1969), there are several ways in which zoning in the upper mantle might take place. One (mineralogical zoning) is based on the ability of material with his hypothetical 'pyrolite' (1 part basalt, 3 parts dunite) composition to crystallise into four distinct mineral assemblages with different physical properties. Which of these will form at any given time and place will be determined largely by the intersection of geotherms with the assemblages' stability fields; in other words, the assemblage forming will be the one stable at the relevant temperature and pressure. And since temperature and pressure vary with depth in the mantle, so will physical properties. A second possibility is chemical zoning in which "the extensive fractional melting and upward segregation of low-melting components that is inferred to have occurred beneath highly evolved con-

\title{
Recognition of lac initiator
}

Protein synthesis in Escherichia coli begins after the ribosome and transfer $\mathrm{RNA}_{f}{ }^{\text {Met }}$ have bound to a region of the messenger RNA containing the initiator triplet codon. An intriguing question is how the machinery recognises and selects the correct AUG (or GUG) codon out of others that may be fortuitously or otherwise present. Maybe a specific sequence of bases is recognised or maybe there is some aspect of the secondary structure with a recognisable shape.

An approach to this problem is to make use of the fact that initiation complex is stable in the absence of other charged tRNA species so that protein synthesis is prevented from taking place. It has been found that a region of the messenger RNA is protected from attack by endonucleases by the bound ribosome and it is tempting to believe that these protected regions contain the features that are recognised as well as the initiator codon.

The base sequence of several such ribosomal binding sites have already been published for various cistrons of various phages and a possible initiator triplet has invariably been found. In some of these sequences the protected sequence looks as if it could form a region of helical secondary structure with the codon in the loop, and it has been mooted that it is some aspect of this structure that is recognised by the $E$. coli ribosome.

This attractive notion seems to be confounded by work by Maizels (see page 647 of this issue of Nature). She had already reported the sequence of the first 63 bases of the lactose operon messenger RNA transcribed from the CAP-independent UV5 promoter mutant. The first AUG triplet occurs at positions $39-41$ and is followed by 21 bases that code for the first seven amino acids of $\beta$-galactosidase. This suggested that translation might initiate at position 39 and that ribosomes should protect the surrounding region.

Maizels has gone on in her new article to sequence the region of this messenger that is protected by the ribosome in the initiation complex. The results gratifyingly show that the region around position 39 is indeed protected, though the precise length of the protected region depends on the activity and nature of the degrading endonuclease. It next turns out that no obvious secondary structure can be constructed with the codon in the loop.

The next step was to inspect the various ribosome protected regions obtained from the lac messenger and from the phages to see if common sequences are present. From this several generalisations emerge. First, there is always a nonsense triplet within 22 bases of the initiator codon, on the $5^{\prime}$ side; second, seven of the eleven sequences including the lac messenger show the quadruplet AGGA on the 5' side; third, many of the sequences, again including lac, show a sequence PuPuUUUXPu ( $\mathrm{Pu}$ is a purine and $\mathrm{X}$ is usually a purine). The significance of these findings is, however, further confused by the fact that these common subsequences do not occur in the same position relative to the initiator codon or even in the same order.

The question remains as to whether these results provide a tantalising glimpse at the requirements for initiator recognition or whether they are only fortuitous; for the possibility is still open that the binding of the ribosome to the protected region is not the primary event and that the recognition involves some other part of the messenger or even that the recognition process involves some other messenger specific factor.

E. G. RICHARDS 